\title{
EFECTO DEL 1-METILCICLOPROPENO (1-MCP) Y DE UNA PELÍCULA COMESTIBLE SOBRE LA ACTIVIDAD ENZIMÁTICA Y CALIDAD POSCOSECHA DEL MANGO 'ATAULFO'
}

\author{
EFFECT OF 1-METHYLCYCLOPROPENE (1-MCP) AND AN EDIBLE COATING ON ENZYME ACTIVITY AND \\ POSTHARVEST QUALITY OF MANGO 'ATAULFO'
}

\author{
Dolores Muy Rangel ${ }^{1 *}$, Blanca Espinoza Valenzuela ${ }^{1}$, Jorge Siller Cepeda ${ }^{1}$, J. Adriana Sañudo Barajas ${ }^{1}$, \\ Benigno Valdez Torres ${ }^{2}$ y Tomás Osuna Enciso ${ }^{1}$
}

\begin{abstract}
${ }^{1}$ Centro de Investigación en Alimentación y Desarrollo, A. C. Unidad Culiacán. Carr. a El Dorado Km. 5.5. Campo en Diez. Apdo. postal 32-A. 80129, Culiacán, Sinaloa, México. Tel. y Fax 01 (667) 760-5536 Ext 221. ${ }^{2}$ Instituto Tecnológico de Estudios Superiores de Monterrey-Campus Sinaloa. Blv. Pedro Infante 3773 Pte. 80100, Culiacán, Sinaloa, México.
\end{abstract}

\section{RESUMEN}

Por la importancia económica del mango (Mangifera indica L.) cv. 'Ataulfo' en México, en este trabajo se estudió el efecto de 1MCP (1-metilciclopropeno) y de una película comestible (PC) a base de quitosano, sobre variables de calidad y de vida de anaquel del mango 'Ataulfo', así como el efecto del 1-MCP sobre la actividad de enzimas hidrolíticas de la pared celular. Los frutos en madurez fisiológica se dividieron en seis tratamientos: 1) Testigo; 2) PC; 3) 1MCP, $400 \mathrm{~nL} \mathrm{~L}^{-1}$; 4) 1-MCP, $800 \mathrm{~nL} \mathrm{~L}^{-1}$; 5) PC + 1-MCP, $400 \mathrm{~nL}$ $\mathrm{L}^{-1}$; y 6) $\mathrm{PC}+1-\mathrm{MCP}, 800 \mathrm{~nL} \mathrm{~L}^{-1}$. Los frutos se almacenaron $7 \mathrm{~d}$ a $12{ }^{\circ} \mathrm{C}$ y se transfirieron a $2{ }^{\circ} \mathrm{C}$ por $15 \mathrm{~d}$. Se evaluó pérdida de peso, firmeza, pH, acidez titulable y sólidos solubles totales (SST), respiración y actividad enzimática de poligalacturonasa (PG), celulasa $(\mathbf{C x}), \alpha$ - y $\beta$-galactosidasa y $\beta$-glucosidasa. Los tratamientos con 1MCP y PC no redujeron la pérdida de peso de los frutos, pero los tratados con $400 \mathrm{~nL} \mathrm{~L}^{-1}$ de $1-\mathrm{MCP}$ mantuvieron mayor firmeza. Luego de $3 \mathrm{~d}$ de almacenamiento los frutos testigo y PC perdieron más de $50 \%$ de la firmeza, y a los 9 d perdieron su calidad comercial. No se observaron cambios en $\mathrm{pH}$ y SST por efecto de los tratamientos en los frutos almacenados por $12 \mathrm{~d}$. La respiración disminuyó en todos los frutos tratados con relación al testigo; los frutos con 1-MCP + PC retrasaron en $3 \mathrm{~d}$ el máximo climatérico. La actividad de PG y Cx se incrementó durante la maduración y la primera fue mayor en frutos testigo que en los tratados, mientras que la máxima actividad de glicosidasas se observó en el día 6. En los frutos tratados con 1-MCP se notó una reducción en las actividades de PG y Cx.

Palabras clave: Mangifera indica, ablandamiento, maduración, 1metilciclopropeno.

\section{SUMMARY}

Because of the economic impact of mango (Mangifera indica $\mathbf{L}$.) fruit cv. 'Ataulfo' in México, the effect of 1-MCP (1methylcyclopropene) and of a chitosan-based edible coat (EC) on the quality parameters and shelf life of 'Ataulfo' mango, and the effect of 1-MCP on the hydrolytic activity of cell wall enzymes were studied in this work. Physiologically mature fruits were assigned into six treatments: 1) Control; 2) EC; 3) 1-MCP, $400 \mathrm{~nL} \mathrm{~L}^{-1}$; 4) 1-MCP, 800 $\mathrm{nL} \mathrm{L}^{-1}$; 5) EC + 1-MCP, $400 \mathrm{~nL} \mathrm{~L}^{-1}$; and 6) $\mathrm{EC}+1-\mathrm{MCP}, 800 \mathrm{~nL}$ $\mathrm{L}^{-1}$. Mangos were first stored for $7 \mathrm{~d}$ at $12{ }^{\circ} \mathrm{C}$ and then transferred to $20{ }^{\circ} \mathrm{C}$ for $15 \mathrm{~d}$. Weight loss, firmness, $\mathrm{pH}$, titratable acidity, total soluble solids (TSS), respiration rate and the activities of polygalacturonase (PG), cellulase $(\mathrm{Cx}), \alpha$ - and $\beta$-galactosidase and $\beta$ glucosidase were evaluated. 1-MCP and EC did not reduce weight loss in mango, but fruits treated with $400 \mathrm{~nL} \mathrm{~L}^{-1}$ of 1-MCP maintained the highest values of firmness throughout storage, whereas the control and EC treated fruits had already lost more than $50 \%$ of their initial firmness $3 \mathrm{~d}$ after treatment, and the commercial quality was lost by day 9. Fruits did not show any significant change in $\mathbf{p H}$ and TSS by the imposed treatments during $12 \mathrm{~d}$ at storage condition. Respiration decreased in all treated fruits as compared to controls; 1-MCP + EC treated fruits delayed their climacteric peaks by $3 \mathrm{~d}$. PG and $\mathrm{Cx}$ activities increased during fruit ripening, and PG activity was higher in control compared to fruits from other treatments. The highest activity of both glycosidases was observed on day 6 . In fruits treated with 1-MCP, a reduction of PG and Cx activities was observed.

Index words: Mangifera indica, softening, maturity, 1methylcyclopropene.

\section{INTRODUCCIÓN}

El mango (Mangifera indica L.) es uno de los frutos tropicales más apreciados en el mundo y de alta importancia económica. Los principales países productores son India, China, Tailandia y México (FAO, 2007). En 2006 la producción de mango en México fue de 1.7 millones de toneladas aproximadamente (SAGARPA, 2007), distribuidas principalmente en los cultivares 'Manila' (431 405 t), 'Ataulfo' (308 544 t), 'Haden' (232 451 t), 'Tommy Atkins' (220 243 t), criollos (166 448 t), 'Kent' (164 827 t) y 'Keitt' (81 147 t) (SIAP, 2008). La calidad del mango mexicano ubica al país como el principal exportador a nivel mundial con $21 \%$ y a Estados Unidos como su principal comprador. El mango 'Ataulfo' con denominación de origen "Mango Ataulfo del Soconusco Chiapas" (IMPI, 2003), ha aumentado su importancia económica (Luna et 
al., 2006), pero su comercialización se ha restringido por la rápida pérdida de calidad de sus frutos debido a la maduración acelerada, pérdida de firmeza y desarrollo de enfermedades. Aunque el mango no produce cantidades considerables de etileno (hormona de la maduración), es altamente sensible a su acción (Kader, 2002); al ser expuesto a trazas de etileno acelera la disminución de la firmeza, la cual se atribuye principalmente a cambios en la pared celular por acción de enzimas hidrolíticas (Seymour y Gross, 1996).

En mango la reducción de la firmeza se asocia con la actividad de las enzimas poligalacturonasa (PG: E.C.3.2.1.15) y celulasa (Cx: E.C.3.2.1.4), donde la despolimerización de las pectinas ha sido ampliamente relacionada con la actividad de la PG (Roe y Bruemmer, 1981; El-Zoghbi, 1994;). Sin embargo, otros estudios muestran que estas enzimas no son determinantes en el ablandamiento, sino que éste se debe a los cambios de las pectinas con la acción de la $\beta$-galactosidasa (E.C.3.2.1.23) y exo-galactanasas (E.C.3.2.1.67) (Huber et al., 2001). Además, se ha reportado que durante la maduración del mango ocurre una pérdida de azúcares (galactosa) de la pared celular (Mitcham y McDonald, 1992).

Una alternativa para reducir el efecto del etileno sobre la maduración de frutos climatéricos como el mango, es el uso del 1-metilciclopropeno (1-MCP) cuya efectividad depende del material de estudio, tiempo de exposición, concentración y temperatura de aplicación. El 1-MCP induce efectos favorables en firmeza, respiración, producción de etileno, degradación de clorofila y desarrollo de enfermedades en frutas y hortalizas (Blankenship y Dole, 2003). Jeong y Huber (2004) señalaron que el 1-MCP redujo la actividad de $\alpha$-galactosidasa (E.C.3.2.1.22), $\beta$ galactosidasa y PG en aguacate (Persea americana Mill.). Sin embargo, no existe evidencia sobre un efecto reductor en la transpiración de los frutos. Por ello, la combinación de una película comestible con 1-MCP podría ser una alternativa apropiada de manejo poscosecha.

El uso de recubrimientos comestibles modifica la atmósfera interna de gases y reduce el metabolismo y transpiración de frutas y hortalizas (Baldwin et al., 1999; Carrillo-López et al., 2000). Un recubrimiento comestible utilizado es el quitosano, polisacárido extraído de exoesqueletos de cangrejo, camarón y langosta (Shepherd et al., 1997), el cual ha mostrado ser efectivo para prolongar la vida poscosecha de litchi (Litchi chinensis Sonn.) (Jiang et al., 2005). El objetivo del presente trabajo fue determinar el efecto del 1-MCP y de una película comestible elaborada con quitosano, en variables de calidad y vida de anaquel de mango 'Ataulfo', así como el efecto de la aplicación de 1-MCP sobre la actividad de enzimas hidrolíticas de la pared celular.

\section{MATERIALES Y MÉTODOS}

Los mangos 'Ataulfo' se cosecharon en madurez fisiológica (frutos con cambio de color externo de verde intenso a verde pálido y color interno de blanco-verdoso a amarillo claro) durante el ciclo 2005 (Junio-Julio), en una huerta comercial ubicada en Navolato, Sinaloa, México. Se seleccionaron 684 frutos que se lavaron y dividieron en seis grupos iguales; a cada lote se le aplicó uno de los siguientes tratamientos de recubrimiento del fruto: 1) Testigo (sin tratamiento químico); 2) PC (Película comestible) formulada a $12{ }^{\circ}$ Brix y diluida a $1.2^{\circ} \mathrm{Brix}$; 3) 1-MCP (1metilciclopropeno), $400 \mathrm{~nL} \mathrm{~L}^{-1}$; 4) 1-MCP, $800 \mathrm{~nL} \mathrm{~L}^{-1}$; 5) $\mathrm{PC}+1-\mathrm{MCP}, 400 \mathrm{~nL} \mathrm{~L}^{-1}$; y 6) $\mathrm{PC}+1-\mathrm{MCP}, 800 \mathrm{~nL}$ $\mathrm{L}^{-1}$. La PC se aplicó por aspersión $(2 \mathrm{~mL})$ y el 1-MCP a 400 y $800 \mathrm{~nL} \mathrm{~L}^{-1}$ en forma de gas durante $24 \mathrm{~h}$ a $12{ }^{\circ} \mathrm{C}$. Los frutos se almacenaron a $12{ }^{\circ} \mathrm{C}$ por $7 \mathrm{~d}$ y después fueron transferidos a $20{ }^{\circ} \mathrm{C}$ por $15 \mathrm{~d}$; ambos tratamientos se emplearon para simular las condiciones de transporte y mercadeo, respectivamente. Las variables de calidad se evaluaron después de aplicar el 1-MCP, a los 7 d a 12 ${ }^{\circ} \mathrm{C}$, y cada $3 \mathrm{~d}$ a $20^{\circ} \mathrm{C}$ por $15 \mathrm{~d}$. El experimento se hizo por duplicado.

Pérdida de peso. Los frutos se pesaron en una balanza digital Mettler Toledo PR 8002. La tasa de pérdida de peso acumulada se determinó en función del peso inicial y peso final en siete frutos por tratamiento y se reportó como porcentaje (Muy et al., 2004).

Firmeza. La firmeza se determinó por punción expresada en Newtons $(\mathrm{N})$, previa eliminación de la epidermis en dos partes de la zona ecuatorial del fruto (Bourne, 1980). Se utilizó un penetrómetro Chatillon Digital (DFIS-50) con un puntal de $10 \mathrm{~mm}$ de diámetro. Se evaluaron cinco frutos por tratamiento.

Acidez titulable y sólidos solubles totales. Las variables químicas de calidad se determinaron en cinco frutos por tratamiento, según el método de la AOAC (1998). Se utilizó un titulador automático Mettler Toledo modelo DL-21 equipado para indicar de manera directa el $\mathrm{pH}$ y la acidez titulable (porcentaje de ácido cítrico). La concentración de sólidos solubles totales (SST) se determinó en una gota del jugo del fruto colocada en un refractómetro Mettler Toledo modelo RE40D calibrado con agua destilada y se expresó en ${ }^{\circ}$ Brix.

Producción de bióxido de carbono $\left(\mathrm{CO}_{2}\right)$. Los frutos se pesaron y se colocaron en frascos de vidrio herméticos de $3.7 \mathrm{~L}$ adaptados con mangueras de entrada y salida de 
aire y conectados a un tren de respiración con flujo constante de aire libre de $\mathrm{CO}_{2}$. Se tomó $1 \mathrm{~mL}$ de aire de la manguera de salida y se analizó en un cromatógrafo de gases Varian 3300. La producción de $\mathrm{CO}_{2}$ se cuantificó en tres frutos por tratamiento, en $\mathrm{mL} \mathrm{CO}_{2} \mathrm{~kg}^{-1} \mathrm{~h}^{-1}$; como estándar se usó $\mathrm{CO}_{2}$ a $0.52 \%$ en mezcla con nitrógeno (AGA Gas México, S. A. de C. V.), según Báez-Sañudo et al. (1997).

Análisis enzimático. Se cuantificó la actividad de las enzimas poligalacturonasa (PG, E.C.3.2.1.15), carboximetilcelulasa $\quad(\mathrm{Cx}, \quad$ E.C.3.2.1.4), $\alpha$-galactosidasa (E.C.3.2.1.22), $\beta$-galactosidasa (E.C.3.2.1.23) y $\beta$ glucosidasa (E.C.3.2.1.22) en la pulpa de frutos. El extracto proteico de la muestra se obtuvo según Koslanund et al. (2005). De la pulpa de un fruto congelado se tomaron $10 \mathrm{~g}$ de muestra homogenizada, se agregaron $10 \mathrm{~mL}$ de la solución amortiguadora HEPES $(50 \mathrm{mM}, \mathrm{pH} 7)$ que contenía $2 \mathrm{mM}$ DTT y $3 \mathrm{mM}$ de bisulfito de sodio, $2 \mathrm{mM}$ de EDTA, PVPP $1 \%$ (p/v) y de Triton X-100 $0.1 \%$ (v/v). Se homogenizó por 3 min y se centrifugó a 11200 $\mathrm{x} g$ durante $30 \mathrm{~min}$ en una centrífuga Thermo-IEC modelo multiRF. El precipitado se resuspendió en una solución amortiguadora HEPES (50 mM pH 7) que contenía $2 \mathrm{mM}$ de DTT, $3 \mathrm{mM}$ bisulfito de sodio, $2 \mathrm{mM}$ EDTA y $1.5 \mathrm{M}$ $\mathrm{NaCl}$. Posteriormente, se agitó durante $2 \mathrm{~h}$ y se centrifugó a 13000 x $g$ durante $30 \mathrm{~min}$. El sobrenadante se desaló en membranas de diálisis MWCO de 8000 Da con una solución de acetato de sodio $(50 \mathrm{mM} \mathrm{pH} \mathrm{5)} \mathrm{durante}$ $48 \mathrm{~h}$, con cambio de solución a las $24 \mathrm{~h}$. El extracto enzimático se concentró en polientilenglicol (PM 15 000-20 $000)$ y se filtró en malla Miracloth ${ }^{\circledR}$. El extracto concentrado se utilizó para la actividad de hidrolasas. Todo el proceso de extracción se efectuó entre $2-4{ }^{\circ} \mathrm{C}$ en cuatro frutos por tratamiento y tiempo.

Actividad enzimática. Se evaluó solamente en frutos tratados con 1-MCP y en el testigo, ya que los tratados con $\mathrm{PC}$ y sus combinaciones (PC $+1-\mathrm{MCP})$ no tuvieron una respuesta significativa en retrasar el ablandamiento de frutos durante el almacenamiento. La actividad de PG y Cx se determinó por separado al incubar a $\quad 37^{\circ} \mathrm{C}, 1$ $\mathrm{mL}$ de extracto proteico y $2 \mathrm{~mL}$ de ácido poligalacturónico $0.2 \%(\mathrm{p} / \mathrm{v})$ para $\mathrm{PG}$ y $2 \mathrm{~mL}$ de carboximetilcelulosa $0.2 \%(\mathrm{p} / \mathrm{v})$ para $\mathrm{Cx}$, ambas a $\mathrm{pH}$ 5. A distintos tiempos se tomaron alícuotas de $100 \mu \mathrm{L}$ y se agregó $1 \mathrm{~mL}$ de solución amortiguadora de boratos $(0.1 \mathrm{M}, \mathrm{pH}$ 9) y $100 \mu \mathrm{L}$ de 2-cianoacetamida $1 \%(\mathrm{p} / \mathrm{v})$; luego se aplicó ebullición por 10 min y se midió la absorbancia a $276 \mathrm{~nm}$ en un espectrofotómetro Cary 1-E (Varian Inc). Para PG la liberación de grupos reductores se determinó con base en una curva patrón de ácido galacturónico $(0 \mathrm{a} 40 \mu \mathrm{g})$ y su actividad se expresó en unidades de liberación de ácido galac- turónico $\left(1 \mathrm{nmol} \mathrm{mg} \mathrm{mg}^{-1}\right.$ proteína $\left.\mathrm{min}^{-1}\right)$. Para Cx se utilizó una curva patrón de glucosa $(0$ a $80 \mu \mathrm{g})$ y la actividad se expresó en unidades de liberación de glucosa $\left(1 \mathrm{nmol} \mathrm{mg}^{-1}\right.$ proteína $\mathrm{min}^{-1}$ ) (Gross, 1982).

Para las galactosidasas se incubaron $500 \mu \mathrm{L}$ de extracto y $500 \mu \mathrm{L}$ del $p$-nitrofenil glicósido correspondiente: $p$ nitrofenil $\alpha$-galactopiranósido, $p$-nitrofenil $\beta$-galactopiranósido y $p$-nitrofenil $\beta$-glucopiranósido para $\alpha$ galactosidasa, $\beta$-galactosidasa y $\beta$-glucosidasa, respectivamente. La reacción se detuvo con $1 \mathrm{~mL}$ de hidróxido de amonio $7 \%(\mathrm{v} / \mathrm{v})$ y se midió la absorbancia a $420 \mathrm{~nm}$ en un espectrofotómetro Varian Cary 1-E. La actividad de galactosidasas se determinó con base en una curva patrón de $p$-nitrofenol $(0$ a $40 \mu \mathrm{g})$ y se expresó en unidades de liberación de $p$-nitrofenol $\left(1 \mathrm{nmol} \mathrm{mg}{ }^{-1}\right.$ proteína $\left.\min ^{-1}\right)$. El contenido de proteína se analizó según Bradford (1976), con albúmina de suero bovino como estándar (Bio-Rad).

Análisis estadístico. El experimento se estableció bajo un diseño completamente al azar con dos factores (tratamiento químico y tiempo) para las variables destructivas (firmeza, $\mathrm{pH}$, acidez titulable, sólidos solubles totales, $\mathrm{PG}, \mathrm{Cx}, \alpha$ - y $\beta$-galactosidasa y $\beta$-glucosidasa), mientras que para las variables no destructivas (pérdida de peso, color y respiración) se utilizó un arreglo de tres factores (tratamiento químico, fruto y tiempo). El factor fruto se anidó en tratamiento químico y el factor tiempo fue de medidas repetidas. El factor tratamiento químico con seis niveles [1) Testigo; 2) PC; 3) 1-MCP, $400 \mathrm{~nL} \mathrm{~L}^{-1}$; 4) 1MCP, $800 \mathrm{~nL} \mathrm{~L}^{-1}$; 5) PC + 1-MCP, $400 \mathrm{~nL} \mathrm{~L}^{-1}$; y 6) PC $+1-\mathrm{MCP}, 800 \mathrm{~nL} \mathrm{~L}^{-1}$ y el factor tiempo con seis niveles $(0,3,6,8,12$ y $15 \mathrm{~d})$. Los efectos de interés fueron: tratamiento químico, tiempo y la interacción tratamiento químico x tiempo. Se hicieron los análisis de varianza, y cuando hubo efectos significativos se aplicó la prueba de comparación de medias de Tukey $(\alpha=0.05)$, mediante el programa estadístico MINITAB (2004) versión 14.0.

\section{RESULTADOS Y DISCUSIÓN}

\section{Pérdida de peso}

Dado a que las variables de calidad evaluadas no mostraron cambios considerables cuando el fruto se almacenó por $7 \mathrm{~d} \mathrm{a} 12{ }^{\circ} \mathrm{C}$ (simulación del tiempo de transporte), se omitieron estos resultados por ser similares a los del día cero a $20{ }^{\circ} \mathrm{C}$, y por ello aquí se reporta solamente el comportamiento del fruto durante simulación de mercadeo $\left(20^{\circ} \mathrm{C}\right)$.

Los mangos tratados con 1-MCP presentaron diferencias significativas en la pérdida de peso después de $6 \mathrm{~d}$ de 
almacenamiento, pero la máxima diferencia alcanzada a los $12 \mathrm{~d}$ fue de $0.5 \%$ y no se reflejó en la calidad de los frutos. La aplicación de la PC no fue efectiva para reducir la pérdida de peso de los mangos en comparación con los tratados con 1-MCP y testigo (Cuadro 1). Esto sugiere que la efectividad de una película comestible depende de las características del fruto (tipo de cutícula y velocidad de transpiración), composición y condiciones de almacenamiento, como propusieron Amarante y Banks (2001).

Baldwin et al. (1999) tampoco observaron una clara diferencia entre mangos 'Tommy Atkins' tratados con Nature Seal ${ }^{\circledR}$ (celulosa) y los frutos testigo. Para ambos resultados se puede considerar lo señalado por Hagenmaier y Shaw (1992), en el sentido de que las películas hechas con carbohidratos poseen características hidrofílicas que permiten la permeabilidad del vapor de agua de manera similar a los frutos no cubiertos.

Los frutos de mandarina (Citrus reticulata L.) tratados con 1-MCP no mostraron diferencias en la pérdida de peso con los frutos sin tratamiento (Laamim et al., 2005), contrario a lo observado en aguacate (Jeong et al., 2002). Esto indica que la efectividad del 1-MCP se relaciona con la fisiología de cada especie, como afirmaron Blankenship y Dole (2003).

De manera general, los mangos 'Ataulfo' presentaron una pérdida de peso superior a $8.5 \%$ durante el almacenamiento, lo que hace necesario el estudio de otras alternativas que ayuden a reducir la transpiración del fruto de forma más efectiva.

\section{Firmeza}

El 1-MCP redujo la pérdida de firmeza de los mangos durante los primeros $9 \mathrm{~d}$ de almacenamiento, pero no cuando se combinó con PC. Los frutos tratados con 1MCP a $400 \mathrm{~nL} \mathrm{~L}^{-1}$ fueron $40 \%$ más firmes durante los 3, 6 y 9 d de almacenamiento que los frutos testigo (Figura 1), lo que podría ser una alternativa poscosecha para mantener la firmeza de los frutos. Luego de $3 \mathrm{~d}$ de almacenamiento los frutos testigo y los tratados con la PC perdieron más de $50 \%$ de firmeza, y a los $10 \mathrm{~d}$ también perdieron su calidad comercial. Mitcham y McDonald (1992) asociaron la pérdida de calidad del mango con valores de firmeza menores a $20 \mathrm{~N}$.

Fue evidente el efecto del $400 \mathrm{~nL} \mathrm{~L}^{-1}$ de 1-MCP para mantener la firmeza de los frutos por mayor tiempo (Figura 1), ya que este compuesto químico retrasa la acción del etileno y los cambios asociados con la maduración del fruto (Sisler y Serek, 1997). Similarmente, Pelayo et al.
(2003) encontraron que los plátanos (Musa paradisiaca L.) tratados con 1-MCP $\left(1000 \mathrm{~nL} \mathrm{~L}^{-1}\right)$ tuvieron mayor firmeza que los testigos, lo que podría incrementar su vida poscosecha. Esto es aún más importante en mangos debido a que la firmeza es determinante para su calidad (Mitcham y McDonald, 1992).

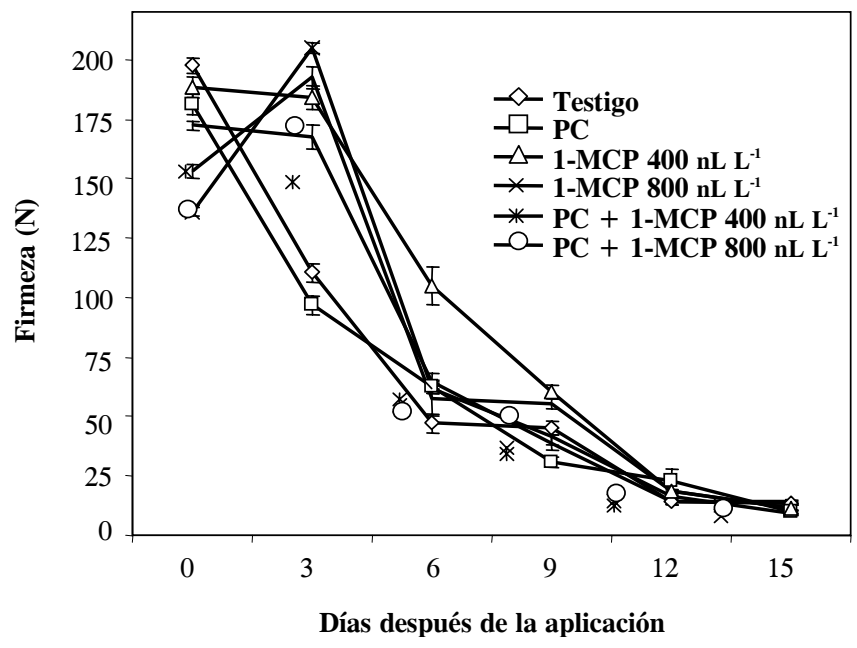

Figura 1. Efecto del 1-MCP y de una película comestible (PC) sobre la firmeza de frutos de mango 'Ataulfo' almacenados a $2{ }^{\circ} \mathrm{C}$. Los datos son media \pm error estándar $(n=5)$.

\section{pH, acidez titulable y sólidos solubles totales (SST)}

Al día cero los mangos mantuvieron valores de $\mathrm{pH}$ de 2.7 y de SST de $10{ }^{\circ}$ Brix, valores que en promedio se incrementaron a $\mathrm{pH}=4.5$ y $16.5^{\circ}$ Brix al final del almacenamiento (Cuadro 1). En acidez titulable se observó una disminución de 2.3 a $0.56 \%$ de ácido cítrico, donde los frutos tratados con 1-MCP $800 \mathrm{~nL} \mathrm{~L}^{-1}$ con y sin PC mantuvieron estadísticamente $(\mathrm{P} \leq 0.05)$ la mayor acidez con respecto al resto de los frutos, sin que hubiera diferencia entre ellos (Cuadro 1). Este comportamiento posiblemente se deba a un retraso ligero en la actividad metabólica (Figura 2) relacionada con velocidad de maduración de los frutos por efecto del 1-MCP. Al igual que en este estudio, Hofman et al. (2001) encontraron una mayor retención de acidez titulable en mangos 'Kensington Pride' tratados con 1-MCP, que asociaron con una maduración más lenta de los frutos. Los altos valores iniciales de acidez encontrados en este estudio son característicos de mangos 'Ataulfo' (Osuna-García et al., 2002); sin embargo, durante la maduración la acidez se pierde hasta alcanzar valores similares a los mangos 'Haden' y 'Kent' maduros (Carrillo-López et al. 2000; Zamora-Cienfuegos et al., 2004). 
Cuadro 1. Efecto del 1-MCP y de la película comestible (PC) sobre el pH, acidez titulable, sólidos solubles totales y pérdida de peso en frutos de mango 'Ataulfo' almacenados a $20{ }^{\circ} \mathrm{C}$.

\begin{tabular}{|c|c|c|c|c|c|c|c|c|c|c|c|c|}
\hline \multirow[b]{3}{*}{ Tratamiento } & \multicolumn{12}{|c|}{ Días a $20^{\circ} \mathrm{C}$} \\
\hline & 0 & 6 & 12 & 0 & 6 & 12 & 0 & 6 & 12 & 0 & 6 & 12 \\
\hline & \multicolumn{3}{|c|}{$\mathrm{pH}^{\dagger}$} & \multicolumn{3}{|c|}{ 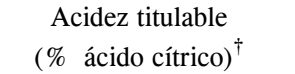 } & \multicolumn{3}{|c|}{$\begin{array}{l}\text { Sólidos solubles totales } \\
\qquad\left({ }^{\circ} \mathrm{Brix}\right)^{\dagger}{ }^{\dagger}\end{array}$} & \multicolumn{3}{|c|}{$\begin{array}{l}\text { Pérdida de peso } \\
(\%)^{\dagger \dagger}\end{array}$} \\
\hline Testigo & $2.6 \mathrm{a}^{\dagger \dagger \dagger}$ & $3.2 \mathrm{a}$ & $4.9 \mathrm{a}$ & $2.7 \mathrm{a}$ & $2.1 \mathrm{a}$ & $0.4 \mathrm{~b}$ & $12.2 \mathrm{a}$ & $15.0 \mathrm{a}$ & $16.9 \mathrm{a}$ & $2.5 \mathrm{a}$ & $4.8 \mathrm{a}$ & $9.1 \mathrm{a}$ \\
\hline PC & $2.6 \mathrm{a}$ & $3.2 \mathrm{a}$ & $4.4 \mathrm{a}$ & $2.2 \mathrm{~b}$ & $2.1 \mathrm{a}$ & $0.5 \mathrm{~b}$ & $8.9 \mathrm{~b}$ & $13.5 \mathrm{~b}$ & $15.6 \mathrm{a}$ & $2.2 \mathrm{a}$ & $5.1 \mathrm{a}$ & $9.4 \mathrm{a}$ \\
\hline 1-MCP $400 \mathrm{~nL} \mathrm{~L}^{-1}$ & $2.7 \mathrm{a}$ & $3.1 \mathrm{a}$ & $4.8 \mathrm{a}$ & $2.4 \mathrm{~b}$ & $2.3 \mathrm{a}$ & $0.4 \mathrm{~b}$ & $11.4 \mathrm{a}$ & $13.5 \mathrm{~b}$ & $15.7 \mathrm{a}$ & $2.3 \mathrm{a}$ & $4.7 \mathrm{~b}$ & $8.9 \mathrm{~b}$ \\
\hline 1-MCP $800 \mathrm{~nL} \mathrm{~L}^{-1}$ & $2.7 \mathrm{a}$ & $3.4 \mathrm{a}$ & $4.7 \mathrm{a}$ & $2.2 \mathrm{~b}$ & $1.9 \mathrm{~b}$ & $0.6 \mathrm{a}$ & $11.1 \mathrm{a}$ & $14.8 \mathrm{a}$ & $15.9 \mathrm{a}$ & $2.6 \mathrm{a}$ & $5.0 \mathrm{a}$ & $9.2 \mathrm{a}$ \\
\hline $\mathrm{PC}+1-\mathrm{MCP} 400 \mathrm{~nL} \mathrm{~L}^{-1}$ & $2.9 \mathrm{a}$ & $3.1 \mathrm{a}$ & $4.4 \mathrm{a}$ & $2.2 \mathrm{~b}$ & $2.2 \mathrm{~b}$ & $0.5 \mathrm{~b}$ & $7.9 \mathrm{~b}$ & $14.4 \mathrm{a}$ & $18.4 \mathrm{a}$ & $2.3 \mathrm{a}$ & $4.4 \mathrm{~b}$ & $9.4 \mathrm{a}$ \\
\hline $\mathrm{PC}+1-\mathrm{MCP} 800 \mathrm{~nL} \mathrm{~L}^{-1}$ & $2.8 \mathrm{a}$ & $2.9 \mathrm{a}$ & $4.1 \mathrm{a}$ & $2.5 \mathrm{a}$ & $2.3 \mathrm{a}$ & $0.9 \mathrm{a}$ & $7.8 \mathrm{~b}$ & $12.7 \mathrm{~b}$ & $16.3 \mathrm{a}$ & $2.4 \mathrm{a}$ & $4.5 \mathrm{~b}$ & $9.5 \mathrm{a}$ \\
\hline
\end{tabular}

${ }^{\dagger}$ Media de 5 frutos. ${ }^{\dagger \dagger}$ Media de 7 frutos. ${ }^{\dagger \dagger}$ Medias con letras iguales por variable y día no son estadísticamente diferentes (Tukey, 0.05).

\section{Producción de $\mathrm{CO}_{2}$}

El comportamiento respiratorio de los mangos correspondió a frutos climatéricos (Figura 2), igual que lo reportado por Montalvo et al. (2007). La máxima actividad en los frutos testigo se observó a los 9 d de almacenamiento con $116 \mathrm{~mL} \mathrm{CO} 2 \mathrm{~kg}^{-1} \mathrm{~h}^{-1}$, seguido por los tratados con PC y $1-\mathrm{MCP}$ a 400 y $800 \mathrm{~nL} \mathrm{~L}^{-1}$. En cambio, en los frutos con $\mathrm{PC}+1-\mathrm{MCP}$ la mayor actividad se registró a los $12 \mathrm{~d}$; todos los frutos tratados, con excepción de la $\mathrm{PC}$, mostraron en promedio una reducción en la velocidad de respiración de más de $40 \%$ con relación al testigo, lo cual se pudo relacionar con el efecto del 1-MCP para retrasar la actividad metabólica de los frutos y su velocidad de maduración, al igual que lo reportado por Blankenship y Dole (2003).

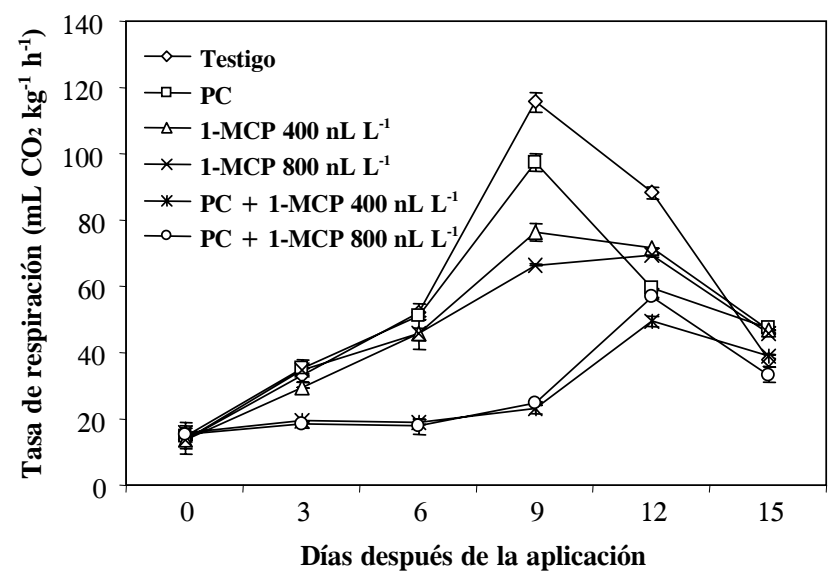

Figura 2. Efecto del 1-MCP y de una película comestible (PC) sobre la tasa de respiración de frutos de mango 'Ataulfo' almacenados a $20{ }^{\circ} \mathrm{C}$. Los datos son media \pm error estándar $(n=3)$.

Similar a este comportamiento, la tasa respiratoria en frutos de plátano (Musa spp.) (Pelayo et al., 2003) y manzana 'Golden Delicious' [Malus sylvestris var. domestica (Borkh.) Mansf.] (Argenta et al., 2007) fue menor en frutos tratados con 1-MCP que en el testigo. Así mismo, el uso de películas comestibles elaboradas con carbohidratos o carnauba en mangos 'Tommy Atkins' retrasó la velocidad de respiración debido al menor intercambio de gases entre el fruto y el medio, y la mayor vida poscosecha de los frutos se asoció con un retraso en la actividad metabólica y la transpiración (Baldwin et al., 1999).

\section{Actividad enzimática}

Al inicio del estudio todos los mangos mostraron una baja actividad de la poligalacturonasa (PG), < 10 nmoles AGA mg prot ${ }^{-1} \mathrm{~min}^{-1}$, la cual se incrementó cuatro veces en los frutos testigo a los $6 \mathrm{~d}$ de almacenamiento, mientras que los tratados con $1-\mathrm{MCP}$ a 400 y $800 \mathrm{~nL} \mathrm{~L}^{-1}$ permanecieron sin cambios significativos $(\mathrm{P}>0.05)$ (Figura $3 \mathrm{~A})$. Este comportamiento se podría relacionar con los mayores valores de firmeza registrados en los frutos tratados con 1-MCP a $400 \mathrm{~nL} \mathrm{~L}^{-1}$ (Figura 1). Sin embargo, a los $12 \mathrm{~d}$ de almacenamiento la actividad enzimática se incrementó en los frutos de los tres tratamientos, sin diferencia $(\mathrm{P}>0.05)$ entre el testigo y los tratados con 400 $\mathrm{nL} \mathrm{L} \mathrm{L}^{-1}$ de 1-MCP. Solamente los frutos tratados con 1MCP $800 \mathrm{~nL} \mathrm{~L}^{-1}$ quedaron en promedio con 17 unidades de actividad por abajo del resto de los frutos (Figura 3A).

El incremento en la actividad de la PG en los frutos a los $12 \mathrm{~d}$ de almacenamiento se atribuye a su proceso natural de maduración, ya que la PG participa en el ablandamiento de los frutos (El-Zoghbi, 1994). No obstante que el 1-MCP actúa de forma irreversible sobre el sitio de acción del etileno, es posible que el fruto haya desarrollado nuevos receptores de etileno capaces de inducir la maduración de los frutos, como señalaron Blankenship y Dole (2003).

La actividad de celulasa (Cx) mostró un comportamiento similar a la PG (Figura 3B), al empezar el día cero con una actividad muy baja en todos los frutos y lograr un incremento $(\mathrm{P} \leq 0.05)$ a los $6 \mathrm{~d}$ en los frutos testigo y en los tratados con 1-MCP a $800 \mathrm{~nL} \mathrm{~L}^{-1}$. La diferencia 
$(\mathrm{P} \leq 0.05)$ en la actividad de la $\mathrm{Cx}$ entre los frutos tratados con 1-MCP a 400 y los tratados con $800 \mathrm{~nL} \mathrm{~L}^{-1}$ registrada a los $6 \mathrm{~d}$ de almacenamiento, se puede asociar con los valores de firmeza de los frutos (Figura 1), ya que a menor actividad de la enzima se registra una mayor firmeza. Posteriormente, la actividad de la Cx continuó incrementando hasta el día 12 , sin diferencia entre los frutos testigo y los tratados con 1-MCP (Figura 3B).

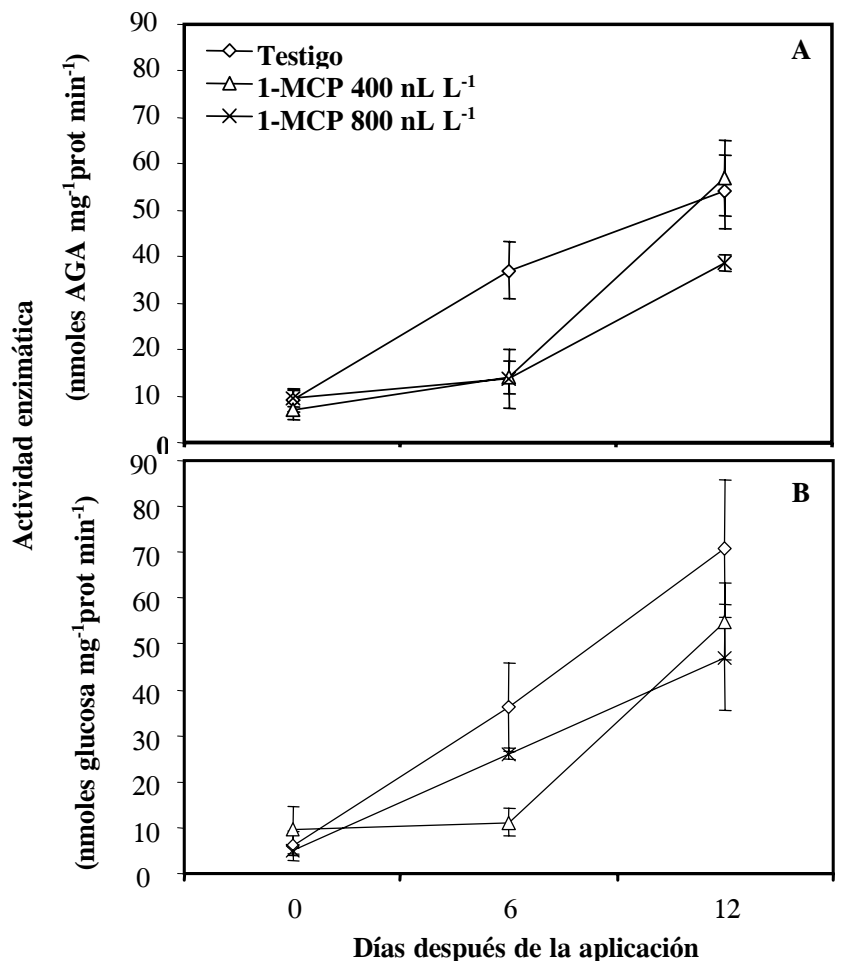

Figura 3. Efecto del 1-MCP sobre la actividad de poligalacturonasa (A) y celulasa (B) en frutos de mango 'Ataulfo' almacenados a 20 ${ }^{\circ} \mathrm{C}$. Los datos son media \pm error estándar $(n=4)$.

El incremento en la actividad de las enzimas PG y Cx durante la maduración de los frutos coincide con otros investigadores (Abu-Sarra y Abu-Goukh, 1992; Mitcham y McDonald, 1992), quienes señalaron que la degradación de las pectinas y la celulosa presente en los frutos es por efecto de las enzimas PG y $\mathrm{Cx}$, respectivamente, las cuales debilitan la pared celular y la consistencia de los frutos. Sin embargo, Muy et al. (2004) reportaron que la pérdida de textura y la ruptura celular del tejido de mango 'Keitt' permiten la salida del jugo del fruto, característica sensorial atractiva por los consumidores pero que es un indicio de senescencia; este fenómeno es el que se busca retrasar con el uso de 1-MCP. En frutos de aguacate tratados con 1-MCP, la actividad PG fue suprimida los primeros $24 \mathrm{~d}$ de almacenamiento en comparación con los no tratados, y también retrasó los cambios en la textura (Jeong y Huber, 2004).
La actividad de $\alpha$-galactosidasa en los frutos (Cuadro 2) inició con la producción de 30 unidades, para luego aumentar $(\mathrm{P} \leq 0.05)$ a 85 unidades a los $6 \mathrm{~d}$ de almacenamiento, pero sin diferencia significativa entre los frutos testigo y los tratados; a los $12 \mathrm{~d}$ esta actividad se vio reducida. Según Huber et al. (2001), el incremento en la actividad de $\alpha$-galactosidasa se relaciona con una pérdida de firmeza de los frutos y pudiera estar asociada con la degradación de pectinas de la lámina media de las células que favorece la pérdida de adhesividad y consistencia de los frutos.

Al inicio del estudio la actividad de la $\beta$-galactosidasa en los mangos superó en $300 \%$ la de $\alpha$-galactosidasa (Cuadro 2), debido posiblemente a que los componentes de la pared celular de los frutos de mango son más ricos en galactanos (Prasanna et al., 2003). Se observó un incremento significativo en la actividad de $\beta$-galactosidasa en los frutos tratados con $800 \mathrm{~nL} \mathrm{~L}^{-1}$ de 1-MCP a los $6 \mathrm{~d}$, seguido de un decremento a los $12 \mathrm{~d}$. La mayor actividad de esta enzima en los frutos tratados con 1-MCP se podría relacionar al estrés que este químico ocasiona al fruto, seguido de una activación de glicosidasas presentes en la pared celular como mecanismo de reserva, según lo reportado por Prasanna et al. (2005).

Cuadro 2. Efecto del 1-MCP en la actividad de las enzimas $\alpha$ galactosidasa, $\beta$-galactosidasa y $\beta$-glucosidasa, en frutos de mango 'Ataulfo' almacenados a $20{ }^{\circ} \mathrm{C}$.

\begin{tabular}{|c|c|c|c|}
\hline \multirow{2}{*}{ Tratamiento } & \multicolumn{3}{|c|}{ Días después de la aplicación } \\
\hline & 0 & 6 & 12 \\
\hline \multicolumn{4}{|c|}{$\alpha$-galactosidasa (nmoles $p$-nitrofenol $\mathrm{mg}^{-1}$ prot $\left.\min ^{-1}\right)^{\dagger}$} \\
\hline Testigo & $29.7 \mathrm{a}^{\dagger \dagger}$ & $53.9 \mathrm{~b}$ & $32.0 \mathrm{~b}$ \\
\hline 1-MCP $400 \mathrm{~nL} \mathrm{~L}^{-1}$ & $30.9 \mathrm{a}$ & $67.9 \mathrm{ab}$ & $62.0 \mathrm{a}$ \\
\hline 1-MCP $800 \mathrm{~nL} \mathrm{~L}^{-1}$ & $33.6 \mathrm{a}$ & $85.0 \mathrm{a}$ & $39.4 \mathrm{~b}$ \\
\hline \multicolumn{4}{|c|}{$\beta$-galactosidasa (nmoles $p$-nitrofenol $\mathbf{m g}^{-1}$ prot min $\left.^{-1}\right)$} \\
\hline Testigo & $105.4 \mathrm{~b}$ & $127.6 \mathrm{~b}$ & $90.0 \mathrm{~b}$ \\
\hline 1-MCP $400 \mathrm{~nL} \mathrm{~L}^{-1}$ & $130.5 \mathrm{a}$ & $140.7 \mathrm{~b}$ & $138.6 \mathrm{a}$ \\
\hline 1-MCP $800 \mathrm{~nL} \mathrm{~L}^{-1}$ & $100.8 \mathrm{~b}$ & $192.0 \mathrm{a}$ & $120.3 \mathrm{~b}$ \\
\hline \multicolumn{4}{|c|}{$\beta$-glucosidasa (nmoles $p$-nitrofenol $\mathrm{mg}^{-1}$ prot $\mathrm{min}^{-1}$ ) } \\
\hline Testigo & $18.5 \mathrm{a}$ & $73.5 \mathrm{~b}$ & $40.0 \mathrm{~b}$ \\
\hline 1-MCP $400 \mathrm{~nL} \mathrm{~L}^{-1}$ & $20.8 \mathrm{a}$ & $80.1 \mathrm{~b}$ & $74.5 \mathrm{a}$ \\
\hline 1-MCP $800 \mathrm{~nL} \mathrm{~L}^{-1}$ & $20.1 \mathrm{a}$ & $114.0 \mathrm{a}$ & $42.5 \mathrm{~b}$ \\
\hline
\end{tabular}

${ }^{\dagger}$ Media de 4 frutos. ${ }^{\dagger \dagger}$ Medias con letras iguales por día y enzima, no son estadísticamente diferentes (Tukey, 0.05).

La actividad de la $\beta$-glucosidasa (Cuadro 2) mostró un comportamiento similar a la $\alpha$-galactosidasa, con una actividad inicial de 20 unidades y un incremento significativo al día 6. Los frutos tratados con $800 \mathrm{~nL} \mathrm{~L}^{-1}$ de 1-MCP tuvieron valores mayores a 114 unidades, mientras que en los frutos testigo y los tratados con $400 \mathrm{~nL} \mathrm{~L}^{-1}$ de 1 -MCP los valores fueron cercanos a 80 unidades; luego hubo una disminución considerable a los $12 \mathrm{~d}$ de almacenamiento. 
A diferencia de las actividades de PG y $\mathrm{Cx}$, los frutos tratados con 1-MCP presentaron mayor actividad de glicosidasas que los frutos testigo; posiblemente esto se deba a que estas enzimas no son dependientes de la respiración ni de la producción de etileno (maduración del fruto), o bien a que la regeneración de nuevos sitios receptores de etileno en el fruto pudieron desencadenar la acción de estas enzimas, debido al estrés y la baja actividad de PG. Una alta actividad de enzimas degradadoras de galactanos se ha correlacionado con pérdida de residuos de azúcares de las fracciones poliméricas de mango (Prasanna et al., 2003), lo cual se podría asociar con el comportamiento de las enzimas glicosidasas estudiadas en el mango 'Ataulfo'.

\section{CONCLUSIONES}

El 1-metilciclopropeno (1-MCP), la película comestible (PC) y sus combinaciones, no fueron efectivos para retrasar la pérdida de peso en los frutos de mango cv. 'Ataulfo' durante el almacenamiento por $7 \mathrm{~d}$ a $12{ }^{\circ} \mathrm{C}+$ $15 \mathrm{~d}$ a $20^{\circ} \mathrm{C}$. La aplicación de $400 \mathrm{~nL} \mathrm{~L}^{-1}$ de 1-MCP fue el tratamiento que logró reducir la pérdida de firmeza de los frutos durante $9 \mathrm{~d}$ de almacenamiento a $20{ }^{\circ} \mathrm{C}$, posterior al almacenamiento por $7 \mathrm{~d}$ a $10{ }^{\circ} \mathrm{C}$. La aplicación de 1-MCP solo o combinado con PC en los mangos disminuyó la actividad respiratoria de los frutos. La actividad de las enzimas poligalacturonasa y carboximetilcelulasa se redujo en los frutos tratados con 1-MCP en comparación con el testigo, y lo contrario ocurrió con la actividad de las enzimas $\alpha$-galactosidasa, $\beta$-galactosidasa y $\beta$ glucosidasas.

\section{AGRADECIMIENTOS}

A la SAGARPA-CONACYT por el apoyo financiero. Al M. C. Manuel Alonzo Báez-Sañudo, M. C. Rosabel Vélez de la Rocha, M. C. Laura Aracely ContrerasAngulo e Ing. Rosalba Contreras-Martínez, por el apoyo técnico.

\section{BIBLIOGRAFÍA}

Abu-Sarra A F, A A Abu-Goukh (1992) Changes in pectinesterase, polygalacturonase and cellulase activity during mango fruit ripening. J. Hortic. Sci. 67:561-568.

Amarante C, N Banks (2001) Postharvest physiology and quality of coated fruits and vegetables. Hort. Rev. 26:161-234.

AOAC, Association of Official Analytical Chemist (1998) Official Methods of Analysis. 16 th ed. Washington, D.C., USA.

Argenta L C, X Fan, J P Mattheis (2007) Responses of 'Golden delicious' apples to 1-MCP applied in air or water. HortScience 42:1651-1655

Báez-Sañudo M, J Siller-Cepeda, B Heredia, T Portillo, E Araiza, R García, D Muy-Rangel (1997) Fisiología poscosecha de frutos de chicozapote (Achras Sapota L.) durante condiciones de mercadeo. Proc. Interamer. Soc. Trop. Hort. 41:209-214.
Baldwin E A, J K Burns, W Kazokas, J K Brecht, R D Hagenmaier, R J Bender, E Pesis (1999) Effect of two edible coatings with different permeability characteristics on mango (Mangifera indica L.) ripening during storage. Postharv. Biol. Technol. 17:215-226.

Blankenship S, J Dole (2003) 1-methylcyclopropene: a review. Postharv. Biol. Technol. 28:1-25.

Bourne M C (1980) Texture evaluation of horticultural crops. HortScience 15:51-57.

Bradford M M (1976) A rapid and sensitive method for the quantization of microgram quantities of protein utilizing the principle of protein-dye binding. Analyt. Biochem. 72:248-254.

Carrillo-López A, F Ramírez-Bustamante, J B Valdez-Torres, R Rojas Villegas, E M Yahia (2000) Ripening and quality changes in mango fruit as affected by coating with an edible film. J. Food Qlty. 23:479-486.

El-Zoghbi M (1994) Biochemical changes in some tropical fruits during ripening. Food Chem. 49:33-37.

FAO (2007) Food and Agriculture Organization. Agricultural data. Disponible en: http://www.fao.org (25/01/2008).

Gross K C (1982) A rapid and sensitive spectrophotometric method for assaying polygalacturonase using 2-cyanoacetamide. HortScience 17:933-934.

Hagenmaier R D, P E Shaw (1992) Gas permeability of fruit coating waxes. J. Am. Soc. Hort. Sci. 117:105-109.

Hofman P J, M Jobin-Décor, G F Meiburg, A J Macnish, D C Joyce (2001) Ripening and quality responses of avocado, custard apple, mango and papaya fruit to 1-methylcyclopropene. Aust. J. Exp. Agric. 41:567-572.

Huber DJ, Y Karakurt, J Jeong (2001) Pectin degradation in ripening and wounded fruits. R. Bras. Fisiol. Veg. 13:224-241.

IMPI (2003) Instituto Mexicano de la Propiedad Intelectual Industrial. Denominación de origen del mango 'Ataulfo'. Disponible en: http://www.impi.gob.mx (09/10/2008).

Jeong J, D Huber (2004) Suppression of avocado (Persea americana Mill.) fruit softening and changes in cell wall matrix polysaccharides and enzyme activities: differential responses to 1MCP and delayed ethylene application. J. Amer. Soc. Hort. Sci. 129:752-759.

Jeong J, D J Huber, S A Sargent (2002) Influence of 1methylcyclopropene (1-MCP) on ripening and cell-wall matrix polysaccharides of avocado (Persea Americana) fruit. Postharv. Biol. Technol. 25:241-256.

Jiang Y, J Li, W Jiang (2005) Effects of chitosan coating on shelf life of cold-stored litchi fruit at ambient temperature. Food Sci. Technol. 38:757-761.

Kader A A (2002) Postharvest Technology of Horticultural Crops. 3rd ed. University of California, Agriculture and Natural Resources. Publication 3311. USA. pp:41, 515.

Koslanund R, D D Archbold, K W Pomper (2005) Pawpaw (Asimina triloba L.) fruit ripening. II. Activity of selected cell-wall degrading enzymes. J. Amer. Soc. Hort. Sci. 130:643-648.

Laamim M, A Ait-Oubahou, M Benichou (2005) Effect of 1methylcyclopropene on the quality of clementine mandarin fruit at ambient temperature. J. Food Agric. Environ. 3:3436

Luna E G, G M Arévalo, R S Anaya, M A Villegas, R M Acosta, R G Leyva (2006) Calidad de mango 'Ataulfo' sometido a tratamiento hidrotérmico. Rev. Fitotec. Mex. 29:123-128.

MINITAB (2004) Statistical software. Release number 14. Disponible en: www.minitab.com.

Mitcham E J, R E McDonald (1992) Cell wall modification during ripening of "Keitt" and "Tommy Atkins" mango fruit. J. Amer. Soc. Hort. Sci. 117:919-924.

Montalvo E, H S García, B Tovar, M Mata (2007) Application of exogenous ethylene on postharvest ripening of refrigerated 'Ataulfo' mangoes. Food Sci. Technol. 40:1466-1472. 
Muy D, J Siller, J Díaz, B Valdez (2004) Las condiciones de almacenamiento y el encerado afectan el estado hídrico y la calidad de mango. Rev. Fitotec. Mex. 27:201-209.

Osuna-García J A, M L Guzmán-Robles, B Tovar-Gómez, M MataMontes de Oca, V A Vidal-Martínez (2002) Calidad del mango 'Ataulfo' producido en Nayarit, México. Rev. Fitotec. Mex. 25:367-374.

Pelayo C, E V Vilas-Boas, M Benichou, A A Kader (2003) Variability in responses of partially ripe bananas to 1methylcyclopropene. Postharv. Biol. Technol. 28:75-85.

Prasanna V, T N Prabha, R N Tharanathan (2003) Pectic polysaccharides of mango (Mangifera indica $\mathrm{L}$ ): structural studies. J. Sci. Food Agric. 83:1182-1186.

Prasanna V, T N Prabha, R N Tharanathan (2005) Multiple forms of $\beta$-galactosidase from mango (Mangifera indica L Alphonso) fruit pulp. J. Sci. Food Agric. 85:797-803.

Roe B, J H Bruemmer (1981) Changes in pectin substances and enzymes during ripening and storage of 'Keitt' mangos. J. Food Sci. 46:186-189.
SAGARPA, Secretaria de Agricultura Ganadería, Desarrollo Rural, Pesca y Alimentación (2007) Servicio de Información y Estadística Agroalimentaria y Pesquera. Anuario Estadístico de la Producción Agrícola 2006. Disponible en: http://siap.gob.mx (18/01/2008).

Seymour G B, K C Gross (1996) Cell wall disassembly and fruit softening. Postharvest News Info. Info 7: 45N-52N; 62.7:45-52.

Shepherd R, S Reader, A Falshaw (1997) Chitosan functional properties. Glycoconj. J. 14:535-542.

Sistema de Información Agroalimentaria y Pesquera, SIAP (2008) Mango. Avance de Siembra y Cosecha. Disponible en: http://www.siap.sagarpa.gob.mx (01/02/2008).

Sisler E C, M Serek (1997) Inhibitors of ethylene responses in plants at the receptor level: recent developments. Physiol. Plant. 100:577-582.

Zamora-Cienfuegos E, H S García, M Mata-Montes de Oca, B Tovar-Gómez (2004) Aceleración de la maduración en mango 'Kent' refrigerado. Rev. Fitotec. Mex. 27:359-366. 